\title{
Active damping of rotating platforms using Integral Force Feedback
}

\author{
T. Dehaeze ${ }^{1,3}$, C. Collette ${ }^{1,2}$ \\ ${ }^{1}$ Precision Mechatronics Laboratory, \\ University of Liege, Belgium \\ ${ }^{2}$ BEAMS Department, \\ Free University of Brussels, Belgium \\ ${ }^{3}$ European Synchrotron Radiation Facility, \\ Grenoble, France \\ e-mail: thomas.dehaeze@esrf.fr
}

\begin{abstract}
This paper investigates the use of Integral Force Feedback (IFF) for the active damping of rotating mechanical systems. Guaranteed stability, typical benefit of IFF, is lost as soon as the system is rotating due to gyroscopic effects. To overcome this issue, two modifications of the classical IFF control scheme are proposed. The first consists of slightly modifying the control law while the second consists of adding springs in parallel with the force sensors. Conditions for stability and optimal parameters are derived. The results reveal that, despite their different implementations, both modified IFF control scheme have almost identical damping authority on suspension modes.
\end{abstract}

\section{Introduction}

There is an increasing need to reduce the undesirable vibration of many sensitive equipment. A common method to reduce vibration is to mount the sensitive equipment on a suspended platform which attenuates the vibrations above the frequency of the suspension modes. In order to further decrease the residual vibrations, active damping can be used for reducing the magnification of the response in the vicinity of the resonances.

In [1], the Integral Force Feedback (IFF) control scheme has been proposed, where a force sensor, a force actuator and an integral controller are used to directly augment the damping of a mechanical system. When the force sensor is collocated with the actuator, the open-loop transfer function has alternating poles and zeros which facilitate to guarantee the stability of the closed loop system [2].

However, when the platform is rotating, gyroscopic effects alter the system dynamics and IFF cannot be applied as is. The purpose of this paper is to study how the IFF strategy can be adapted to deal with these Gyroscopic effects.

The paper is structured as follows. Section 2 presents a simple model of a rotating suspended platform that will be used throughout this study. Section 3 explains how the unconditional stability of IFF is lost due to Gyroscopic effects induced by the rotation. Section 4 suggests a simple modification of the control law such that damping can be added to the suspension modes in a robust way. Section 5 proposes to add springs in parallel with the force sensors to regain the unconditional stability of IFF. Section 6 compares both proposed modifications to the classical IFF in terms of damping authority and closed-loop system behavior. 


\section{Dynamics of Rotating Platforms}

In order to study how the rotation does affect the use of IFF, a model of a suspended platform on top of a rotating stage is used. Figure 1 represents the model schematically which is the simplest in which gyroscopic forces can be studied.

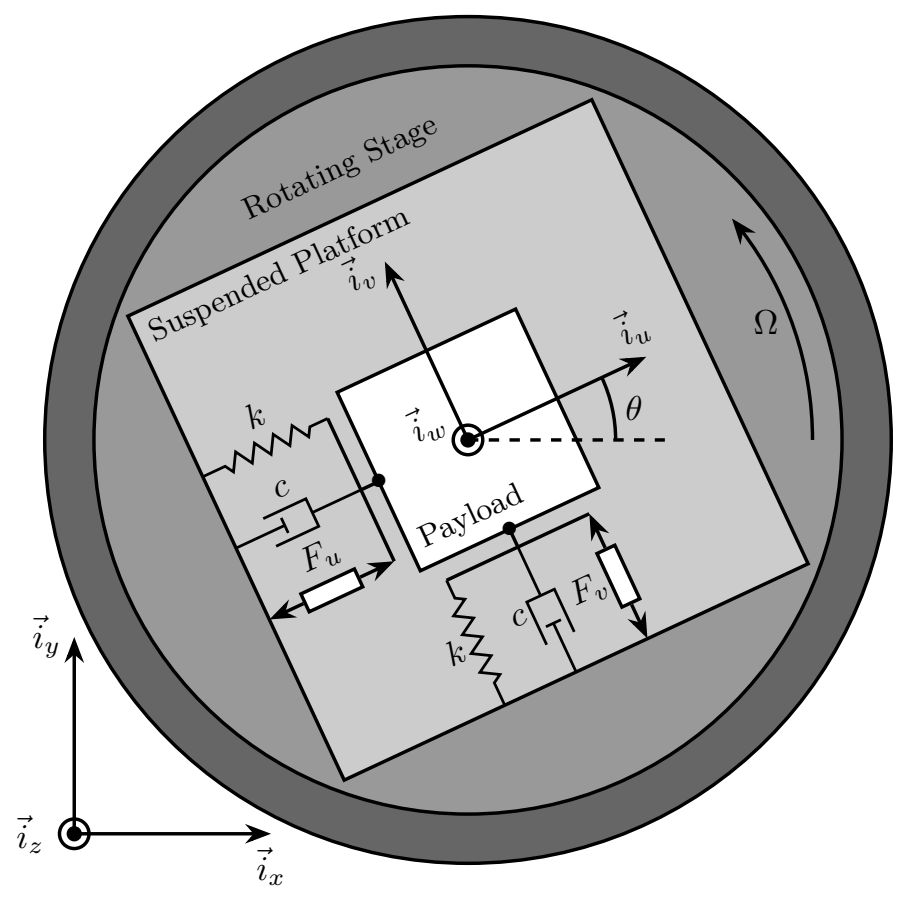

Figure 1: Schematic of the studied System

The rotating stage is supposed to be ideal, meaning it induces a perfect rotation $\theta(t)=\Omega t$ where $\Omega$ is the rotational speed in $\operatorname{rad~s}^{-1}$.

The suspended platform consists of two orthogonal actuators represented by three elements in parallel: a spring with a stiffness $k$ in $\mathrm{N} \mathrm{m}^{-1}$, a dashpot with a damping coefficient $c$ in $\mathrm{N} \mathrm{m}^{-1} \mathrm{~s}$ and an ideal force source $F_{u}, F_{v}$. A payload with a mass $m$ in $\mathrm{kg}$, representing the sensitive equipment, is mounted on the (rotating) suspended platform.

Two reference frames are used: an inertial frame $\left(\vec{i}_{x}, \vec{i}_{y}, \vec{i}_{z}\right)$ and a uniform rotating frame $\left(\vec{i}_{u}, \vec{i}_{v}, \vec{i}_{w}\right)$ rigidly fixed on top of the rotating stage with $\vec{i}_{w}$ aligned with the rotation axis. The position of the payload is represented by $\left(d_{u}, d_{v}, 0\right)$ expressed in the rotating frame.

To obtain the equations of motion for the system represented in Figure 1, the Lagrangian equations are used:

$$
\frac{d}{d t}\left(\frac{\partial L}{\partial \dot{q}_{i}}\right)+\frac{\partial D}{\partial \dot{q}_{i}}-\frac{\partial L}{\partial q_{i}}=Q_{i}
$$

with $L=T-V$ the Lagrangian, $T$ the kinetic coenergy, $V$ the potential energy, $D$ the dissipation function, and $Q_{i}$ the generalized force associated with the generalized variable $\left[\begin{array}{ll}q_{1} & q_{2}\end{array}\right]=\left[\begin{array}{ll}d_{u} & d_{v}\end{array}\right]$. The equation of motion corresponding to the constant rotation in the $\left(\vec{i}_{x}, \vec{i}_{y}\right)$ plane is disregarded as the motion is considered to be imposed by the rotation stage.

$$
\begin{aligned}
& T=\frac{1}{2} m\left(\left(\dot{d}_{u}-\Omega d_{v}\right)^{2}+\left(\dot{d}_{v}+\Omega d_{u}\right)^{2}\right), \quad V=\frac{1}{2} k\left(d_{u}{ }^{2}+d_{v}{ }^{2}\right), \\
& D=\frac{1}{2} c\left(\dot{d}_{u}{ }^{2}+\dot{d}_{v}{ }^{2}\right), \quad Q_{1}=F_{u}, \quad Q_{2}=F_{v}
\end{aligned}
$$


Substituting equations (2) into (1) for both generalized coordinates gives two coupled differential equations

$$
\begin{aligned}
& m \ddot{d}_{u}+c \dot{d}_{u}+\left(k-m \Omega^{2}\right. d_{u}=F_{u}+2 m \Omega \dot{d}_{v} \\
& m \ddot{d}_{v}+c \dot{d}_{v}+(k \underbrace{-m \Omega^{2}}_{\text {Centrif. }}) d_{v}=F_{v} \underbrace{-2 m \Omega \dot{d}_{u}}_{\text {Coriolis }}
\end{aligned}
$$

The uniform rotation of the system induces two Gyroscopic effects as shown in (3):

- Centrifugal forces: that can been seen as added negative stiffness $-m \Omega^{2}$ along $\vec{i}_{u}$ and $\vec{i}_{v}$

- Coriolis Forces: that couples the motion in the two orthogonal directions

To study the dynamics of the system, the differential equations of motions (3) are transformed in the Laplace domain and the $2 \times 2$ transfer function matrix $\boldsymbol{G}_{d}$ from $\left[\begin{array}{ll}F_{u} & F_{v}\end{array}\right]$ to $\left[\begin{array}{ll}d_{u} & d_{v}\end{array}\right]$ is obtained

$$
\begin{aligned}
{\left[\begin{array}{l}
d_{u} \\
d_{v}
\end{array}\right] } & =\boldsymbol{G}_{d}\left[\begin{array}{l}
F_{u} \\
F_{v}
\end{array}\right] \\
\boldsymbol{G}_{d} & =\left[\begin{array}{ll}
\frac{m s^{2}+c s+k-m \Omega^{2}}{\left(m s^{2}+c s+k-m \Omega^{2}\right)^{2}+(2 m \Omega s)^{2}} & \frac{2 m \Omega s}{\left(m s^{2}+c s+k-m \Omega^{2}\right)^{2}+(2 m \Omega s)^{2}} \\
\frac{m s^{2}+c s+k-m \Omega^{2}}{\left(m s^{2}+c s+k-m \Omega^{2}\right)^{2}+(2 m \Omega s)^{2}} & \frac{\left.m s^{2}+c s+k-m \Omega^{2}\right)^{2}+(2 m \Omega s)^{2}}{\left(m s^{2}\right.}
\end{array}\right]
\end{aligned}
$$

To simplify the analysis, the undamped natural frequency $\omega_{0}$ and the damping ratio $\xi$ are used

$$
\omega_{0}=\sqrt{\frac{k}{m}} \text { in rad s}^{-1}, \quad \xi=\frac{c}{2 \sqrt{k m}}
$$

The transfer function matrix $\boldsymbol{G}_{d}$ becomes equal to

$$
\boldsymbol{G}_{d}=\frac{1}{k}\left[\begin{array}{cc}
\frac{\frac{s^{2}}{\omega_{0}{ }^{2}}+2 \xi \frac{s}{\omega_{0}}+1-\frac{\Omega^{2}}{\omega_{0}{ }^{2}}}{\left(\frac{s^{2}}{\omega_{0}^{2}}+2 \xi \frac{s}{\omega_{0}}+1-\frac{\Omega^{2}}{\omega_{0}{ }^{2}}\right)^{2}+\left(2 \frac{\Omega}{\omega_{0}} \frac{s}{\omega_{0}}\right)^{2}} & \frac{2 \frac{\Omega}{\omega_{0}} \frac{s}{\omega_{0}}}{\left(\frac{s^{2}}{\omega_{0}^{2}}+2 \xi \frac{s}{\omega_{0}}+1-\frac{\Omega^{2}}{\omega_{0}{ }^{2}}\right)^{2}+\left(2 \frac{\Omega}{\omega_{0}} \frac{s}{\omega_{0}}\right)^{2}} \\
\frac{-2 \frac{\Omega}{\omega_{0}} \frac{s}{\omega_{0}}}{\left(\frac{s^{2}}{\omega_{0}{ }^{2}}+2 \xi \frac{s}{\omega_{0}}+1-\frac{\Omega^{2}}{\omega_{0}{ }^{2}}\right)^{2}+\left(2 \frac{\Omega}{\omega_{0}} \frac{s}{\omega_{0}}\right)^{2}} & \frac{\frac{s^{2}}{\omega_{0}{ }^{2}}+2 \xi \frac{s}{\omega_{0}}+1-\frac{\Omega^{2}}{\omega_{0}{ }^{2}}}{\left(\frac{s^{2}}{\omega_{0}{ }^{2}}+2 \xi \frac{s}{\omega_{0}}+1-\frac{\Omega^{2}}{\left.\omega_{0}\right)^{2}}\right)^{2}+\left(2 \frac{\Omega}{\omega_{0}} \frac{s}{\omega_{0}}\right)^{2}}
\end{array}\right]
$$

For all further numerical analysis in this study, we consider $\omega_{0}=1 \mathrm{rad} \mathrm{s}^{-1}, k=1 \mathrm{~N} \mathrm{~m}^{-1}$ and $\xi=0.025=$ $2.5 \%$. Even though no system with such parameters will be encountered in practice, conclusions can be drawn relative to these parameters such that they can be generalized to any other set of parameters.

The poles of $\boldsymbol{G}_{d}$ are the complex solutions $p$ of

$$
\left(\frac{p^{2}}{\omega_{0}^{2}}+2 \xi \frac{p}{\omega_{0}}+1-\frac{\Omega^{2}}{\omega_{0}^{2}}\right)^{2}+\left(2 \frac{\Omega}{\omega_{0}} \frac{p}{\omega_{0}}\right)^{2}=0
$$

Supposing small damping $(\xi \ll 1)$, two pairs of complex conjugate poles are obtained:

$$
\begin{aligned}
& p_{+}=-\xi \omega_{0}\left(1+\frac{\Omega}{\omega_{0}}\right) \pm j \omega_{0}\left(1+\frac{\Omega}{\omega_{0}}\right) \\
& p_{-}=-\xi \omega_{0}\left(1-\frac{\Omega}{\omega_{0}}\right) \pm j \omega_{0}\left(1-\frac{\Omega}{\omega_{0}}\right)
\end{aligned}
$$

The real part and complex part of these two pairs of complex conjugate poles are represented in Figure 2 as a function of the rotational speed $\Omega$. As the rotational speed increases, $p_{+}$goes to higher frequencies and $p_{-}$ to lower frequencies. The system becomes unstable for $\Omega>\omega_{0}$ as the real part of $p_{-}$is positive. Physically, the negative stiffness term $-m \Omega^{2}$ induced by centrifugal forces exceeds the spring stiffness $k$. 
In the rest of this study, rotational speeds smaller than the undamped natural frequency of the system are assumed $\left(\Omega<\omega_{0}\right)$.

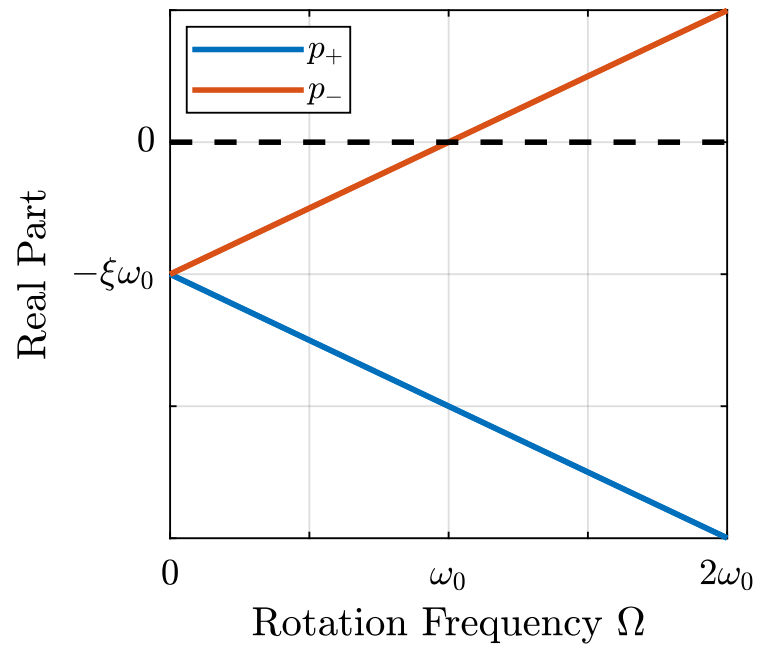

(a) Real Part

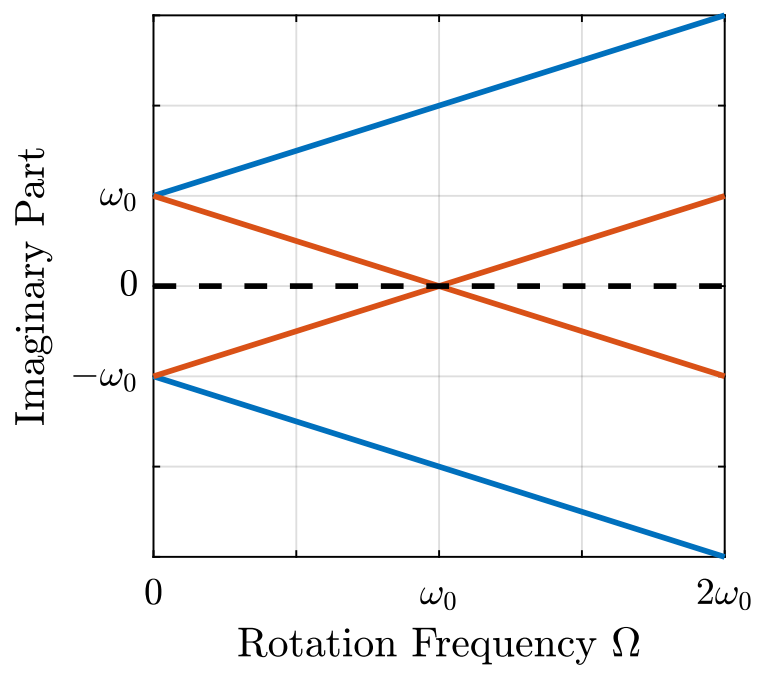

(b) Imaginary Part

Figure 2: Campbell Diagram : Evolution of the complex and real parts of the system's poles as a function of the rotational speed $\Omega$

Looking at the transfer function matrix $\boldsymbol{G}_{d}$ in Eq. (7), one can see that the two diagonal (direct) terms are equal and the two off-diagonal (coupling) terms are opposite. The bode plot of these two terms are shown in Figure 3 for several rotational speeds $\Omega$. These plots confirm the expected behavior: the frequency of the two pairs of complex conjugate poles are further separated as $\Omega$ increases. For $\Omega>\omega_{0}$, the low frequency pair of complex conjugate poles $p_{-}$becomes unstable.

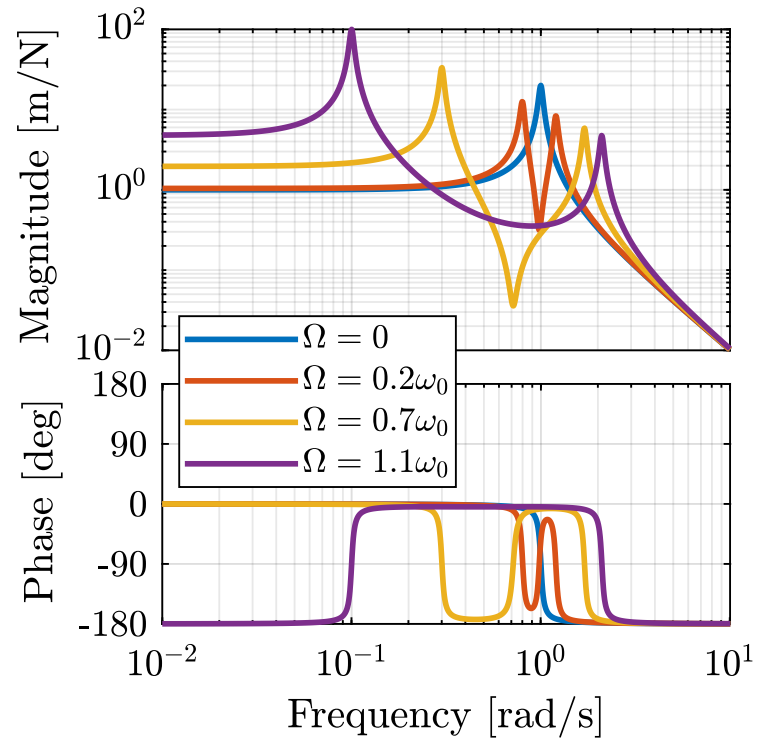

(a) Direct Terms $d_{u} / F_{u}, d_{v} / F_{v}$
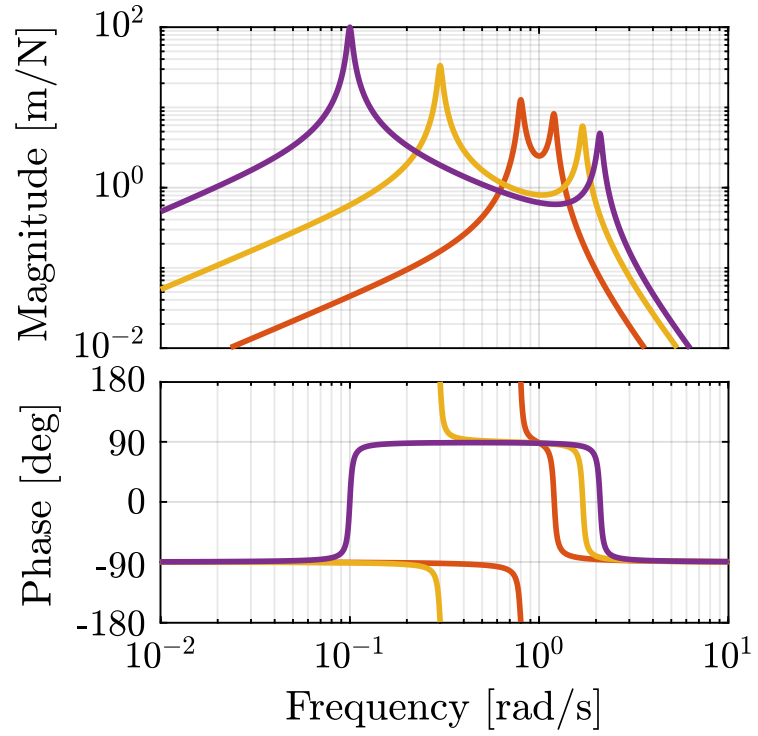

(b) Coupling Terms $d_{v} / F_{u},-d_{u} / F_{v}$

Figure 3: Bode Plots for $\boldsymbol{G}_{d}$ for several rotational speed $\Omega$ 


\section{Decentralized Integral Force Feedback}

In order to apply IFF to the system, force sensors are added in series with the two actuators (Figure 4). As this study focuses on decentralized control, two identical controllers $K_{F}$ are used to feedback each of the sensed force to its associated actuator and no attempt is made to counteract the interactions in the system. The control diagram is schematically shown in Figure 5.

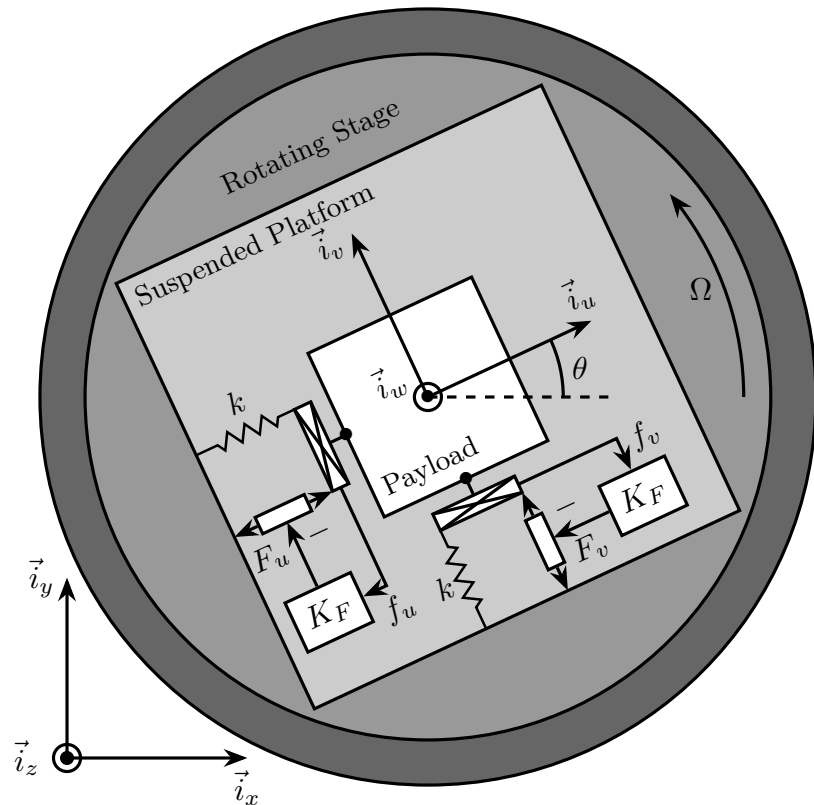

Figure 4: System with added Force Sensor in series with the actuators

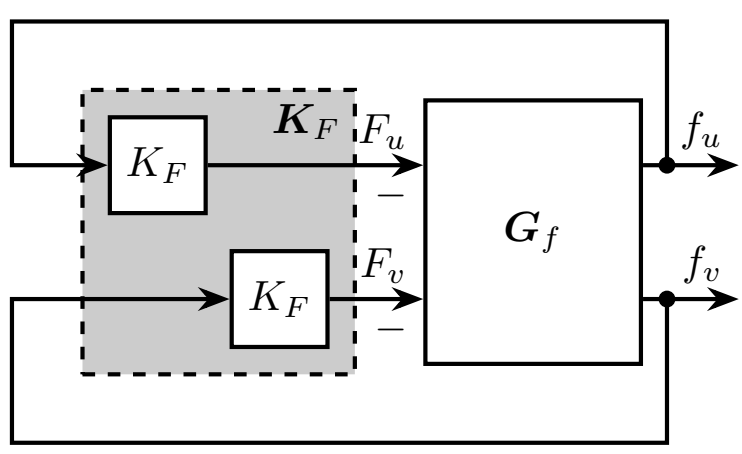

Figure 5: Control Diagram for decentralized IFF

The forces $\left[\begin{array}{ll}f_{u} & f_{v}\end{array}\right]$ measured by the two force sensors represented in Figure 4 are equal to

$$
\left[\begin{array}{l}
f_{u} \\
f_{v}
\end{array}\right]=\left[\begin{array}{l}
F_{u} \\
F_{v}
\end{array}\right]-(c s+k)\left[\begin{array}{l}
d_{u} \\
d_{v}
\end{array}\right]
$$

Inserting (7) into (10) yields

$$
\begin{aligned}
& {\left[\begin{array}{l}
f_{u} \\
f_{v}
\end{array}\right]=\boldsymbol{G}_{f}\left[\begin{array}{l}
F_{u} \\
F_{v}
\end{array}\right]} \\
& \boldsymbol{G}_{f}=\left[\begin{array}{cc}
\frac{\left(\frac{s^{2}}{\omega_{0}^{2}}-\frac{\Omega^{2}}{\omega_{0}^{2}}\right)\left(\frac{s^{2}}{\omega_{0}{ }^{2}}+2 \xi \frac{s}{\omega_{0}}+1-\frac{\Omega^{2}}{\omega_{0}^{2}}\right)+\left(2 \frac{\Omega}{\omega_{0}} \frac{s}{\omega_{0}}\right)^{2}}{\left(\frac{s^{2}}{\omega_{0}{ }^{2}}+2 \xi \frac{s}{\omega_{0}}+1-\frac{\Omega^{2}}{\omega_{0}^{2}}\right)^{2}+\left(2 \frac{\Omega}{\omega_{0}} \frac{s}{\omega_{0}}\right)^{2}} & \frac{-\left(2 \xi \frac{s}{\omega_{0}}+1\right)\left(2 \frac{\Omega}{\omega_{0}} \frac{s}{\omega_{0}}\right)}{\left(\frac{s^{2}}{\omega_{0}^{2}}+2 \xi \frac{s}{\omega_{0}}+1-\frac{\Omega^{2}}{\omega_{0}{ }^{2}}\right)^{2}+\left(2 \frac{\Omega}{\omega_{0}} \frac{s}{\omega_{0}}\right)^{2}} \\
\frac{\left(2 \xi \frac{s}{\omega_{0}}+1\right)\left(2 \frac{\Omega}{\omega_{0}} \frac{s}{\omega_{0}}\right)}{\left(\frac{s^{2}}{\omega_{0}{ }^{2}}+2 \xi \frac{s}{\omega_{0}}+1-\frac{\Omega^{2}}{\omega_{0}{ }^{2}}\right)^{2}+\left(2 \frac{\Omega}{\omega_{0}} \frac{s}{\omega_{0}}\right)^{2}} & \frac{\left(\frac{s^{2}}{\omega_{0}{ }^{2}}-\frac{\Omega^{2}}{\omega_{0}{ }^{2}}\right)\left(\frac{s^{2}}{\omega_{0}{ }^{2}}+2 \xi \frac{s}{\omega_{0}}+1-\frac{\Omega^{2}}{\omega_{0}{ }^{2}}\right)+\left(2 \frac{\Omega}{\omega_{0}} \frac{s}{\omega_{0}}\right)^{2}}{\left(\frac{s^{2}}{\omega_{0}{ }^{2}}+2 \xi \frac{s}{\omega_{0}}+1-\frac{\Omega^{2}}{\omega_{0}{ }^{2}}\right)^{2}+\left(2 \frac{\Omega}{\omega_{0}} \frac{s}{\omega_{0}}\right)^{2}}
\end{array}\right]
\end{aligned}
$$

The zeros of the diagonal terms of $\boldsymbol{G}_{f}$ are equal to (neglecting the damping for simplicity)

$$
\begin{aligned}
& z_{c}= \pm j \omega_{0} \sqrt{\frac{1}{2} \sqrt{8 \frac{\Omega^{2}}{\omega_{0}^{2}}+1}+\frac{\Omega^{2}}{\omega_{0}^{2}}+\frac{1}{2}} \\
& z_{r}= \pm \omega_{0} \sqrt{\frac{1}{2} \sqrt{8 \frac{\Omega^{2}}{\omega_{0}^{2}}+1}-\frac{\Omega^{2}}{\omega_{0}^{2}}-\frac{1}{2}}
\end{aligned}
$$

The frequency of the pair of complex conjugate zeros $z_{c}$ (13a) always lies between the frequency of the two 
pairs of complex conjugate poles $p_{-}$and $p_{+}$(9).

For non-null rotational speeds, two real zeros $z_{r}(13 \mathrm{~b})$ appear in the diagonal terms inducing a non-minimum phase behavior. This can be seen in the Bode plot of the diagonal terms (Figure 6) where the low frequency gain is no longer zero while the phase stays at $180^{\circ}$.

The low frequency gain of $\boldsymbol{G}_{f}$ increases with the rotational speed $\Omega$

$$
\lim _{\omega \rightarrow 0}\left|\boldsymbol{G}_{f}(j \omega)\right|=\left[\begin{array}{cc}
\frac{\Omega^{2}}{\omega_{0}^{2}-\Omega^{2}} & 0 \\
0 & \frac{\Omega^{2}}{\omega_{0}^{2}-\Omega^{2}}
\end{array}\right]
$$

This can be explained as follows: a constant force $F_{u}$ induces a small displacement of the mass $d_{u}=\frac{F_{u}}{k-m \Omega^{2}}$, which increases the centrifugal force $m \Omega^{2} d_{u}=\frac{\Omega^{2}}{\omega_{0}^{2}-\Omega^{2}} F_{u}$ which is then measured by the force sensors.

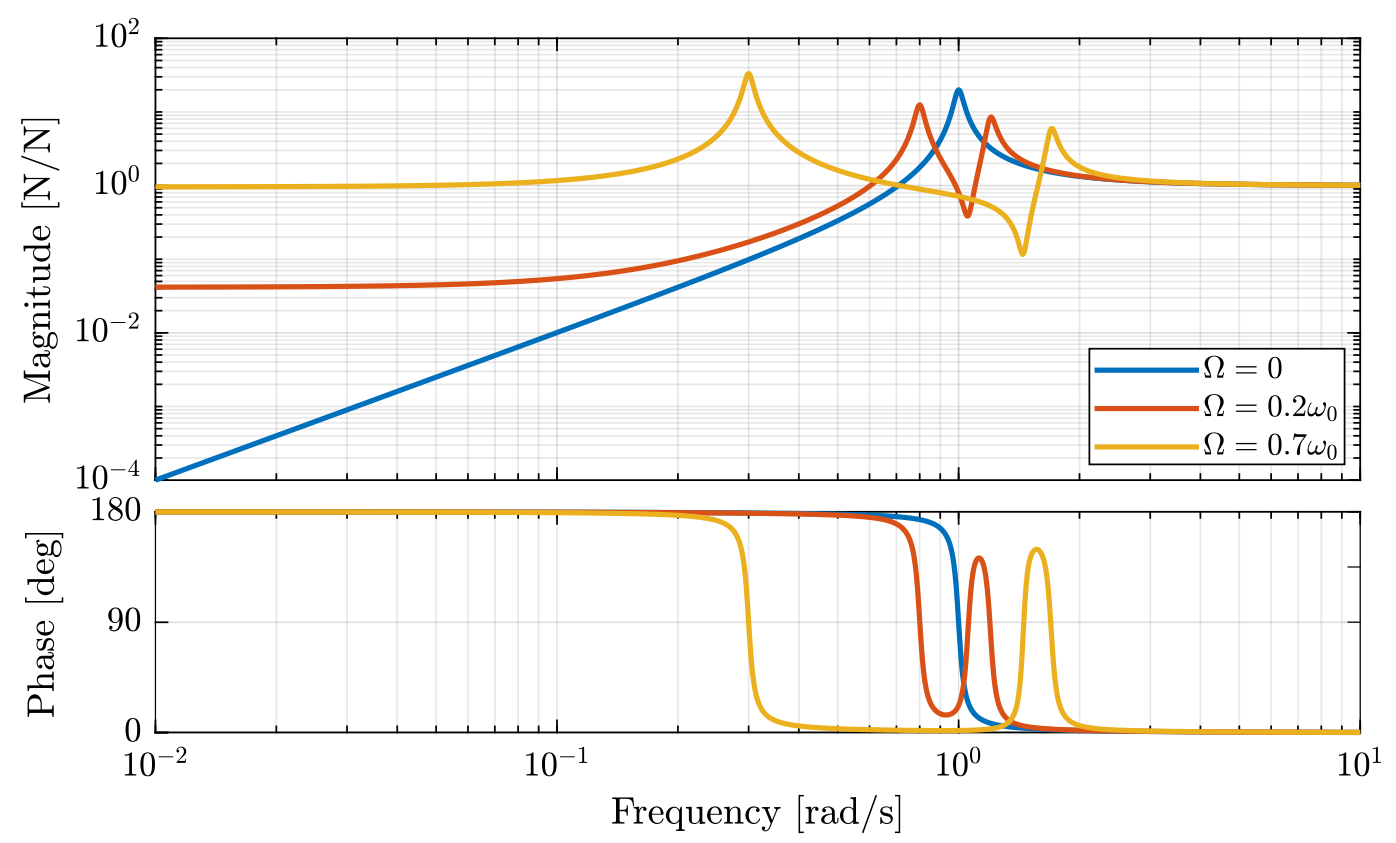

Figure 6: Bode plot of the dynamics from a force actuator to its collocated force sensor $\left(f_{u} / F_{u}, f_{v} / F_{v}\right)$ for several rotational speeds $\Omega$

The two IFF controllers $K_{F}$ consist of a pure integrator

$$
\boldsymbol{K}_{F}(s)=\left[\begin{array}{cc}
K_{F}(s) & 0 \\
0 & K_{F}(s)
\end{array}\right], \quad K_{F}(s)=g \cdot \frac{1}{s}
$$

where $g$ is a scalar representing the gain of the controller.

In order to see how the IFF affects the poles of the closed loop system, a Root Locus plot (Figure 7) is constructed as follows: the poles of the closed-loop system are drawn in the complex plane as the controller gain $g$ varies from 0 to $\infty$ for the two controllers $K_{F}$ simultaneously. As explained in [3, 4], the closed-loop poles start at the open-loop poles (shown by $\mathbf{x}$ ) for $g=0$ and coincide with the transmission zeros (shown by $\mathbf{O}$ ) as $g \rightarrow \infty$. The direction of increasing gain is indicated by arrows

Whereas collocated IFF is usually associated with unconditional stability [5], this property is lost as soon as the rotational speed in non-null due to gyroscopic effects. This can be seen in the Root Locus plot (Figure 7) where the poles corresponding to the controller are bound to the right half plane implying closed-loop system instability.

Physically, this can be explain like so: at low frequency, the loop gain is very large due to the pure integrators in $K_{F}$. The control system is thus canceling the spring forces which makes the suspended platform no able to hold the payload against centrifugal forces, hence the instability. 


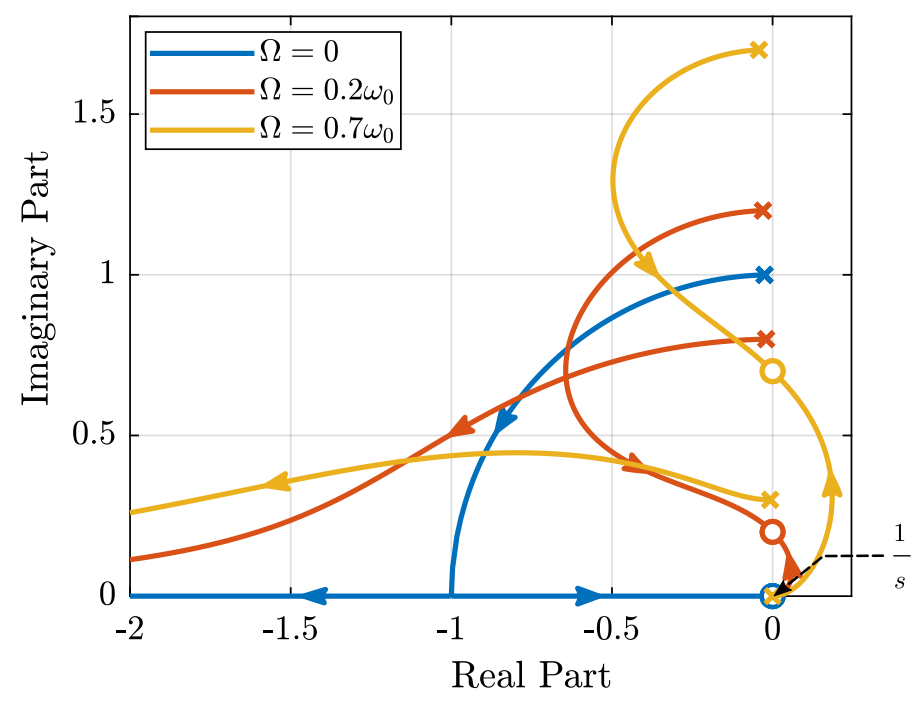

Figure 7: Root Locus: evolution of the closed-loop poles with increasing controller gains $g$

In order to apply decentralized IFF on rotating platforms, two solutions are proposed to deal with this instability problem. The first one consists of slightly modifying the control law (Section 4) while the second one consists of adding springs in parallel with the force sensors (Section 5).

\section{Integral Force Feedback with High Pass Filter}

As was explained in the previous section, the instability comes in part from the high gain at low frequency caused by the pure integrators.

In order to limit this low frequency controller gain, an high pass filter (HPF) can be added to the controller

$$
K_{F}(s)=g \cdot \frac{1}{s} \cdot \underbrace{\frac{s / \omega_{i}}{1+s / \omega_{i}}}_{\mathrm{HPF}}=g \cdot \frac{1}{s+\omega_{i}}
$$

This is equivalent to slightly shifting the controller pole to the left along the real axis.

This modification of the IFF controller is typically done to avoid saturation associated with the pure integrator [5]. This is however not the case in this study as it will become clear in the next section.

The loop gains, $K_{F}(s)$ times the direct dynamics $f_{u} / F_{u}$, with and without the added HPF are shown in Figure 8. The effect of the added HPF limits the low frequency gain as expected.

The Root Loci for the decentralized IFF with and without the HPF are displayed in Figure 9. With the added HPF, the poles of the closed loop system are shown to be stable up to some value of the gain $g_{\max }$

$$
g_{\max }=\omega_{i}\left(\frac{\omega_{0}^{2}}{\Omega^{2}}-1\right)
$$

It is interesting to note that $g_{\max }$ also corresponds to the gain where the low frequency loop gain (Figure 8) reaches one. 

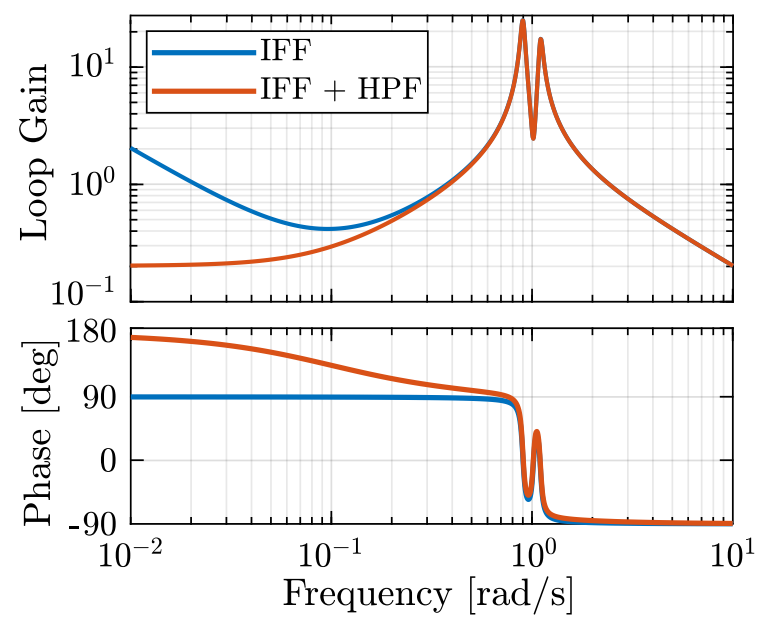

Figure 8: Modification of the loop gain with the added HFP, $g=2, \omega_{i}=0.1 \omega_{0}$ and $\Omega=0.1 \omega_{0}$

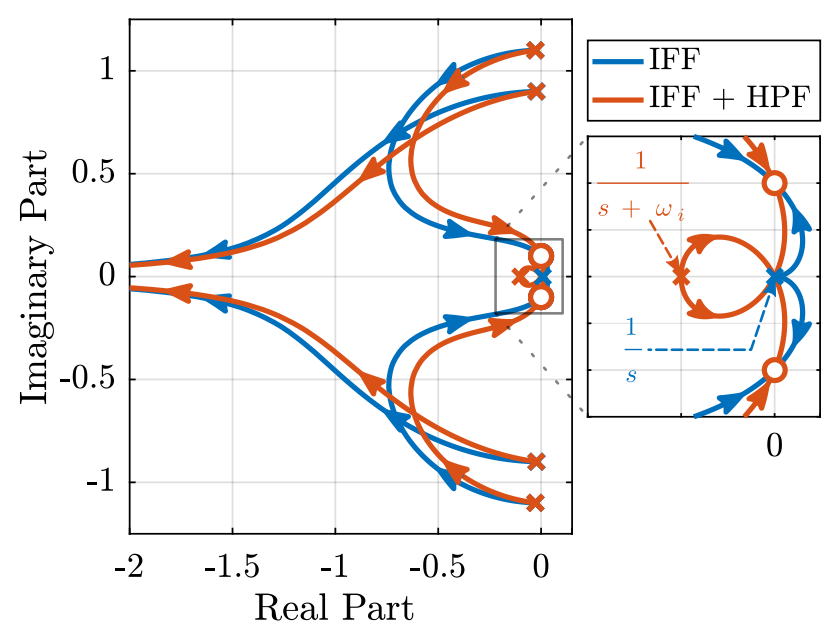

Figure 9: Modification of the Root Locus with the added HPF, $\omega_{i}=0.1 \omega_{0}$ and $\Omega=0.1 \omega_{0}$

Two parameters can be tuned for the modified controller (16): the gain $g$ and the pole's location $\omega_{i}$. The optimal values of $\omega_{i}$ and $g$ are here considered as the values for which the damping of all the closed-loop poles are simultaneously maximized.

In order to visualize how $\omega_{i}$ does affect the attainable damping, the Root Loci for several $\omega_{i}$ are displayed in Figure 10. It is shown that even though small $\omega_{i}$ seem to allow more damping to be added to the suspension modes, the control gain $g$ may be limited to small values due to (17).
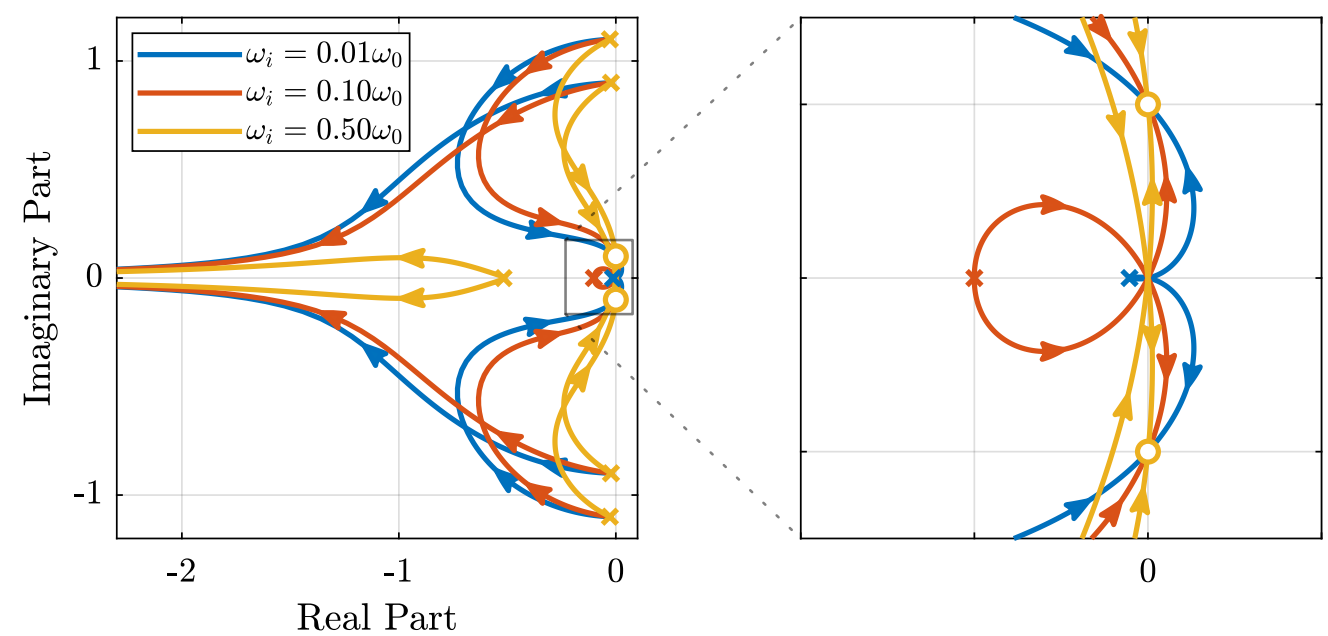

Figure 10: Root Locus for several HPF cut-off frequencies $\omega_{i}, \Omega=0.1 \omega_{0}$

In order to study this trade off, the attainable closed-loop damping ratio $\xi_{\mathrm{cl}}$ is computed as a function of $\omega_{i} / \omega_{0}$. The gain $g_{\mathrm{opt}}$ at which this maximum damping is obtained is also displayed and compared with the gain $g_{\max }$ at which the system becomes unstable (Figure 11).

Three regions can be observed:

- $\omega_{i} / \omega_{0}<0.02$ : the added damping is limited by the maximum allowed control gain $g_{\max }$

- $0.02<\omega_{i} / \omega_{0}<0.2$ : the attainable damping ratio is maximized and is reached for $g \approx 2$

- $0.2<\omega_{i} / \omega_{0}$ : the added damping decreases as $\omega_{i} / \omega_{0}$ increases 


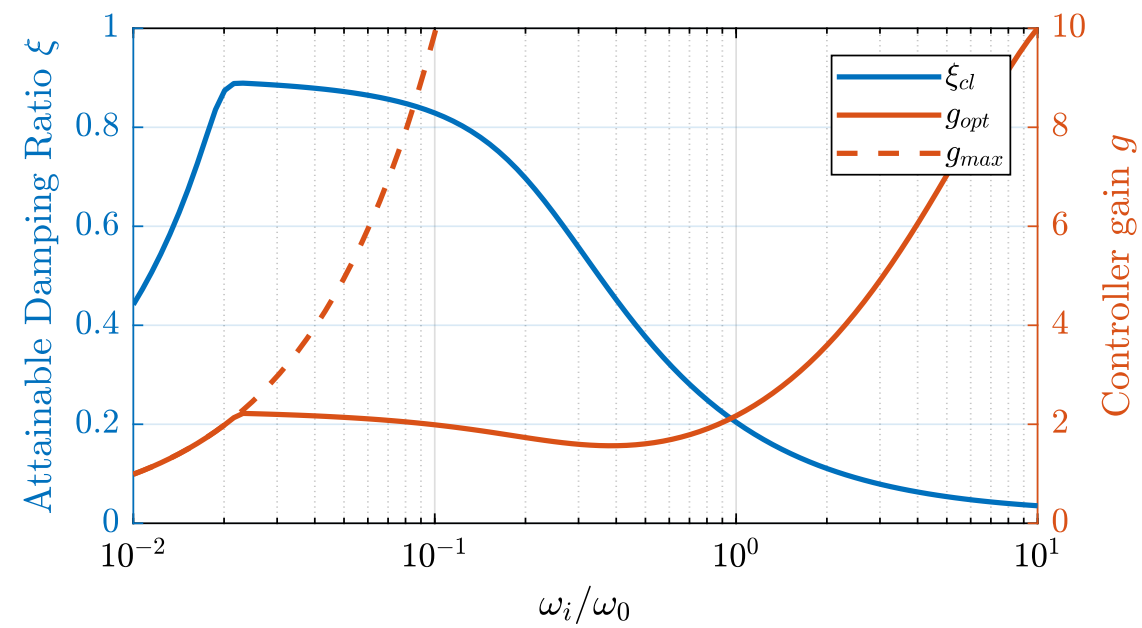

Figure 11: Attainable damping ratio $\xi_{\mathrm{cl}}$ as a function of $\omega_{i} / \omega_{0}$. Corresponding control gain $g_{\mathrm{opt}}$ and $g_{\max }$ are also shown

\section{Integral Force Feedback with Parallel Springs}

In this section additional springs in parallel with the force sensors are added to counteract the negative stiffness induced by the rotation. Such springs are schematically shown in Figure 12 where $k_{a}$ is the stiffness of the actuator and $k_{p}$ the stiffness in parallel with the actuator and force sensor.

Amplified piezoelectric stack actuators can also be used for such purpose where a part of the piezoelectric stack is used as an actuator while the rest is used as a force sensor [6]. The parallel stiffness $k_{p}$ then corresponds to the amplification structure. An example of such system is shown in Figure 13.

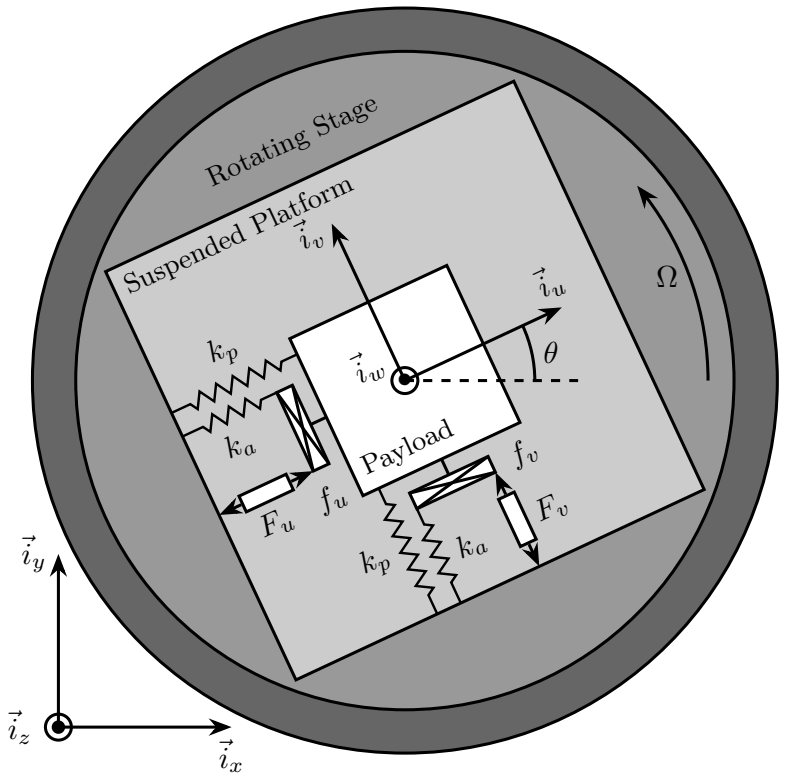

Figure 12: Studied system with additional springs in parallel with the actuators and force sensors

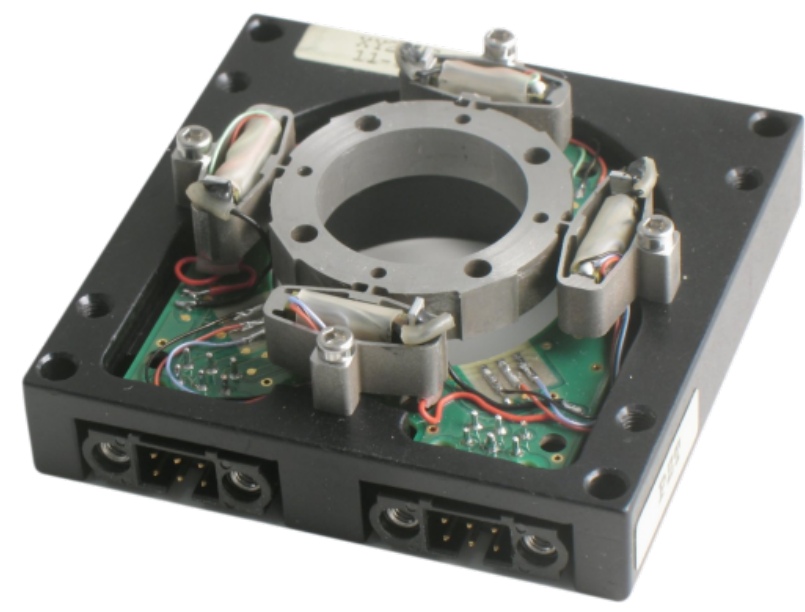

Figure 13: XY Piezoelectric Stage (XY25XS from Cedrat Technology)

The forces $\left[\begin{array}{ll}f_{u} & f_{v}\end{array}\right]$ measured by the two force sensors represented in Figure 12 are equal to

$$
\left[\begin{array}{l}
f_{u} \\
f_{v}
\end{array}\right]=\left[\begin{array}{l}
F_{u} \\
F_{v}
\end{array}\right]-\left(c s+k_{a}\right)\left[\begin{array}{l}
d_{u} \\
d_{v}
\end{array}\right]
$$

In order to keep the overall stiffness $k=k_{a}+k_{p}$ constant, thus not modifying the open-loop poles as $k_{p}$ is 
changed, a scalar parameter $\alpha(0 \leq \alpha<1)$ is defined to describe the fraction of the total stiffness in parallel with the actuator and force sensor

$$
k_{p}=\alpha k, \quad k_{a}=(1-\alpha) k
$$

The equations of motion are derived and transformed in the Laplace domain

$$
\begin{aligned}
& {\left[\begin{array}{l}
f_{u} \\
f_{v}
\end{array}\right]=\boldsymbol{G}_{k}\left[\begin{array}{l}
F_{u} \\
F_{v}
\end{array}\right]} \\
& \boldsymbol{G}_{k}=\left[\begin{array}{cc}
\frac{\left(\frac{s^{2}}{\omega_{0}^{2}}-\frac{\Omega^{2}}{\omega_{0}^{2}}+\alpha\right)\left(\frac{s^{2}}{\omega_{0}^{2}}+2 \xi \frac{s}{\omega_{0}}+1-\frac{\Omega^{2}}{\omega_{0}{ }^{2}}\right)+\left(2 \frac{\Omega}{\omega_{0}} \frac{s}{\omega_{0}}\right)^{2}}{\left(\frac{s^{2}}{\omega_{0}^{2}}+2 \xi \frac{s}{\omega_{0}}+1-\frac{\Omega^{2}}{\omega_{0}{ }^{2}}\right)^{2}+\left(2 \frac{\Omega}{\omega_{0}} \frac{s}{\omega_{0}}\right)^{2}} & \frac{-\left(2 \xi \frac{s}{\omega_{0}}+1-\alpha\right)\left(2 \frac{\Omega}{\omega_{0}} \frac{s}{\omega_{0}}\right)}{\left(\frac{s^{2}}{\omega^{2}}+2 \xi \frac{s}{\omega_{0}}+1-\frac{\Omega^{2}}{\omega_{0}{ }^{2}}\right)^{2}+\left(2 \frac{\Omega}{\omega_{0}} \frac{s}{\omega_{0}}\right)^{2}} \\
\frac{\left(2 \xi \frac{s}{\omega_{0}}+1-\alpha\right)\left(2 \frac{\Omega}{\omega_{0}} \frac{s}{\omega_{0}}\right)}{\left(\frac{s^{2}}{\omega_{0}{ }^{2}}+2 \xi \frac{s}{\omega_{0}}+1-\frac{\Omega^{2}}{\omega_{0}{ }^{2}}\right)^{2}+\left(2 \frac{\Omega}{\omega_{0}} \frac{s}{\omega_{0}}\right)^{2}} & \frac{\left(\frac{s^{2}}{\omega_{0}{ }^{2}}-\frac{\Omega^{2}}{\omega_{0}{ }^{2}}+\alpha\right)\left(\frac{s^{2}}{\omega_{0}{ }^{2}}+2 \xi \frac{s}{\omega_{0}}+1-\frac{\Omega^{2}}{\omega_{0}{ }^{2}}\right)+\left(2 \frac{\Omega}{\omega_{0}} \frac{s}{\omega_{0}}\right)^{2}}{\left(\frac{s^{2}}{\omega_{0}{ }^{2}}+2 \xi \frac{s}{\omega_{0}}+1-\frac{\Omega^{2}}{\omega_{0}{ }^{2}}\right)^{2}+\left(2 \frac{\Omega}{\omega_{0}} \frac{s}{\omega_{0}}\right)^{2}}
\end{array}\right]
\end{aligned}
$$

Comparing $\boldsymbol{G}_{k}$ (21) with $\boldsymbol{G}_{f}$ (12) shows that while the poles of the system are kept the same, the zeros of the diagonal terms have changed. The two real zeros $z_{r}(13 \mathrm{~b})$ that were inducing non-minimum phase behavior are transformed into complex conjugate zeros if the following condition hold

$$
\alpha>\frac{\Omega^{2}}{\omega_{0}^{2}} \Leftrightarrow k_{p}>m \Omega^{2}
$$

Thus, if the added parallel stiffness $k_{p}$ is higher than the negative stiffness induced by centrifugal forces $m \Omega^{2}$, the direct dynamics from actuator to force sensor will show minimum phase behavior. This is confirmed by the Bode plot of the direct dynamics in Figure 14.

Figure 15 shows Root Loci plots for $k_{p}=0, k_{p}<m \Omega^{2}$ and $k_{p}>m \Omega^{2}$ when $K_{F}$ is a pure integrator (15). It is shown that if the added stiffness is higher than the maximum negative stiffness, the poles of the closed-loop system stay in the (stable) right half-plane, and hence the unconditional stability of IFF is recovered.

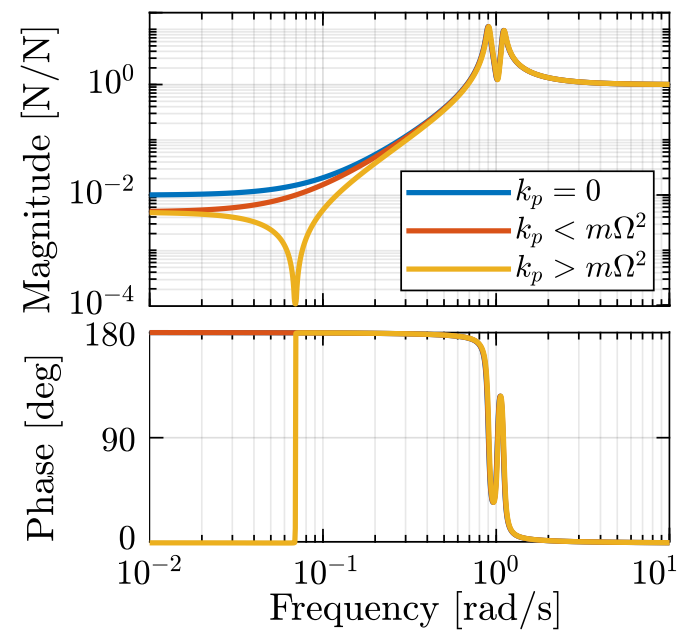

Figure 14: Bode Plot of $f_{u} / F_{u}$ without parallel spring, with parallel springs with stiffness $k_{p}<m \Omega^{2}$ and $k_{p}>m \Omega^{2}, \Omega=0.1 \omega_{0}$

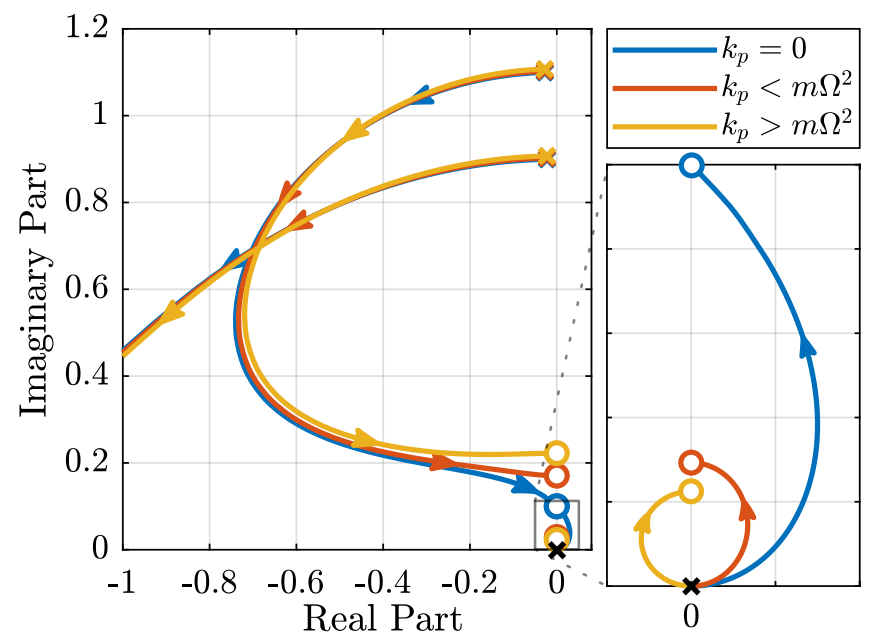

Figure 15: Root Locus for IFF without parallel spring, with parallel springs with stiffness $k_{p}<m \Omega^{2}$ and $k_{p}>$ $m \Omega^{2}, \Omega=0.1 \omega_{0}$

Even though the parallel stiffness $k_{p}$ has no impact on the open-loop poles (as the overall stiffness $k$ stays constant), it has a large impact on the transmission zeros. Moreover, as the attainable damping is generally proportional to the distance between poles and zeros [7], the parallel stiffness $k_{p}$ is foreseen to have a large impact on the attainable damping.

To study this effect, Root Locus plots for several parallel stiffnesses $k_{p}>m \Omega^{2}$ are shown in Figure 16. The frequencies of the transmission zeros of the system are increasing with the parallel stiffness $k_{p}$ and the 
associated attainable damping is reduced. Therefore, even though the parallel stiffness $k_{p}$ should be larger than $m \Omega^{2}$ for stability reasons, it should not be taken too high as this would limit the attainable bandwidth.

This is confirmed in Figure 17 where the attainable closed-loop damping ratio $\xi_{\mathrm{cl}}$ and the associated control gain $g_{\mathrm{opt}}$ are computed as a function of $\alpha$.

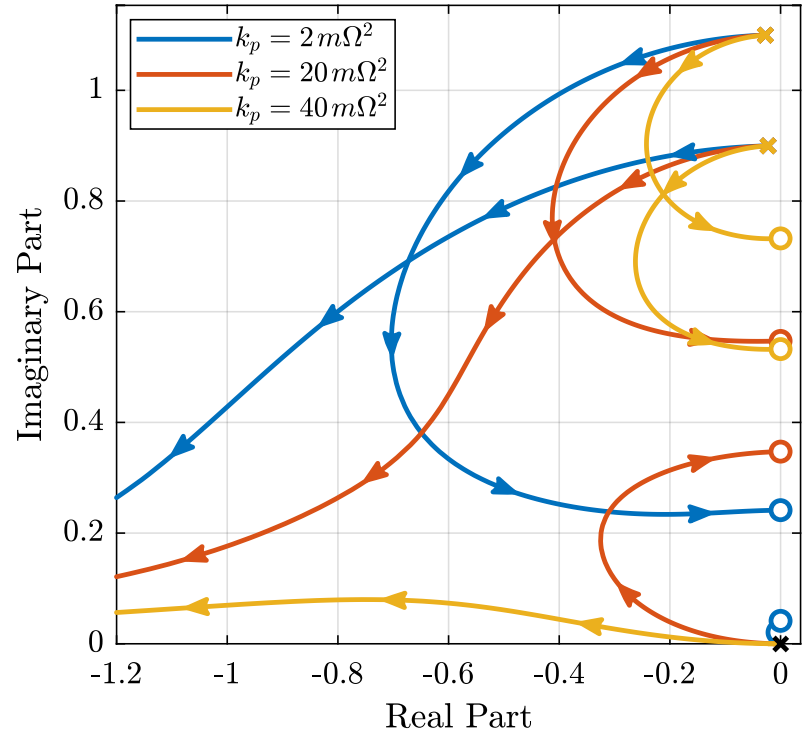

Figure 16: Comparison the Root Locus for three parallel stiffnessses $k_{p}$

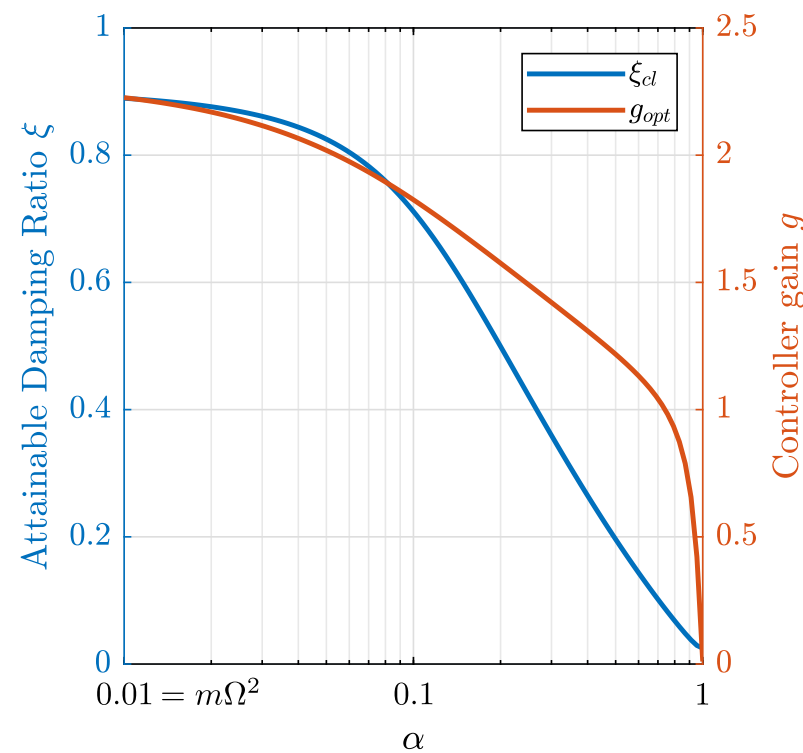

Figure 17: Optimal Damping Ratio $\xi_{\text {opt }}$ and the corresponding optimal gain $g_{\mathrm{opt}}$ as a function of $\alpha$

\section{Comparison and Discussion}

Two modifications to adapt the IFF control strategy to rotating platforms have been proposed in Sections 4 and 5. These two methods are now compared in terms of added damping, closed-loop compliance and transmissibility.

For the following comparisons, the cut-off frequency for the HPF is set to $\omega_{i}=0.1 \omega_{0}$ and the stiffness of the parallel springs is set to $k_{p}=5 m \Omega^{2}$.

Figure 18 shows the Root Loci for the two proposed IFF modifications. While the two pairs of complex conjugate open-loop poles are identical for both techniques, the transmission zeros are not. This means that the closed-loop behavior of both systems will differ when large control gains are used.

One can observe that the closed loop poles of the system with added springs (in red) are bounded to the left half plane implying unconditional stability. This is not the case for the system where the controller is augmented with an HPF (in blue).

It is interesting to note that the maximum added damping is very similar for both techniques and is reached for the same control gain $g_{\text {opt }} \approx 2 \omega_{0}$.

The two proposed techniques are now compared in terms of closed-loop transmissibility and compliance.

The transmissibility is defined as the transfer function from the displacement of the rotating stage to the displacement of the payload. It is used to characterize how much vibration is transmitted through the suspended platform to the payload.

The compliance describes the displacement response of the payload to external forces applied to it. This is a useful metric when disturbances are directly applied to the payload.

The two techniques are also compared with passive damping (Figure 1) where the damping coefficient $c$ is tuned to critically damp the resonance when the rotating speed is null.

$$
c_{\text {crit }}=2 \sqrt{k m}
$$




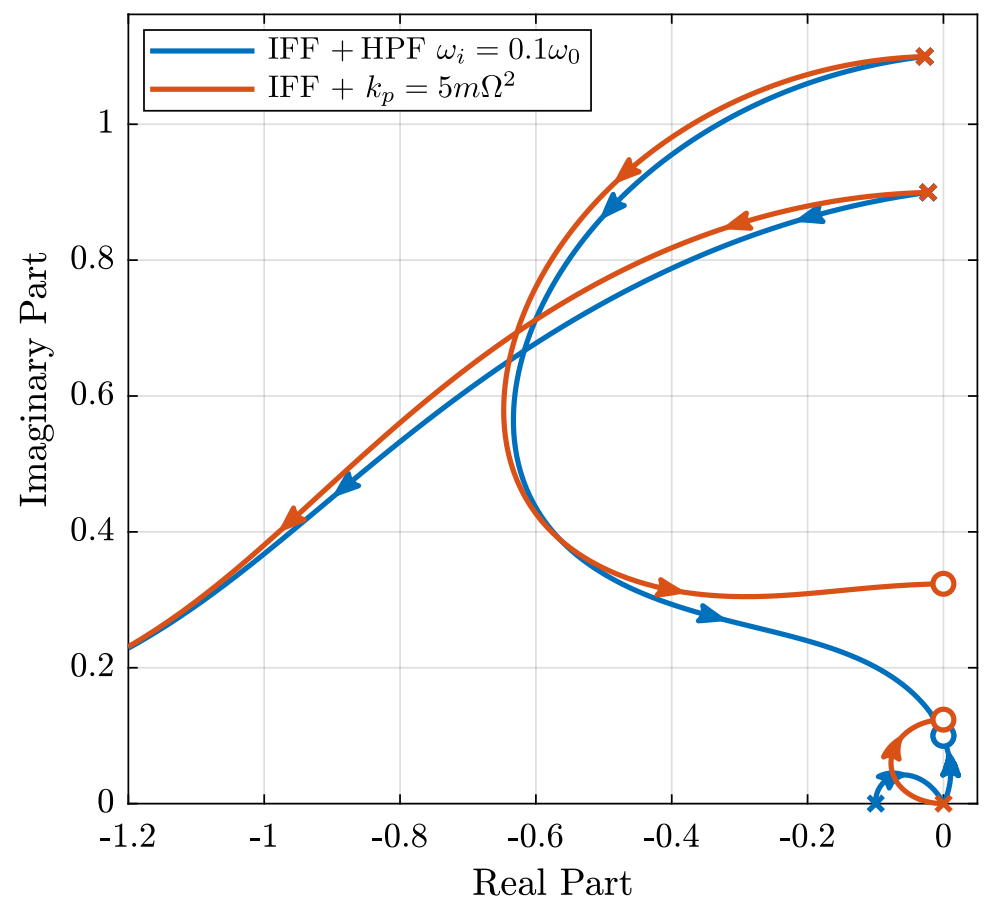

Figure 18: Root Locus for the two proposed modifications of decentralized IFF, $\Omega=0.1 \omega_{0}$

Very similar results are obtained for the two proposed IFF modifications in terms of transmissibility (Figure 19a) and compliance (Figure 19b). It is also confirmed that these two techniques can significantly damp the suspension modes.

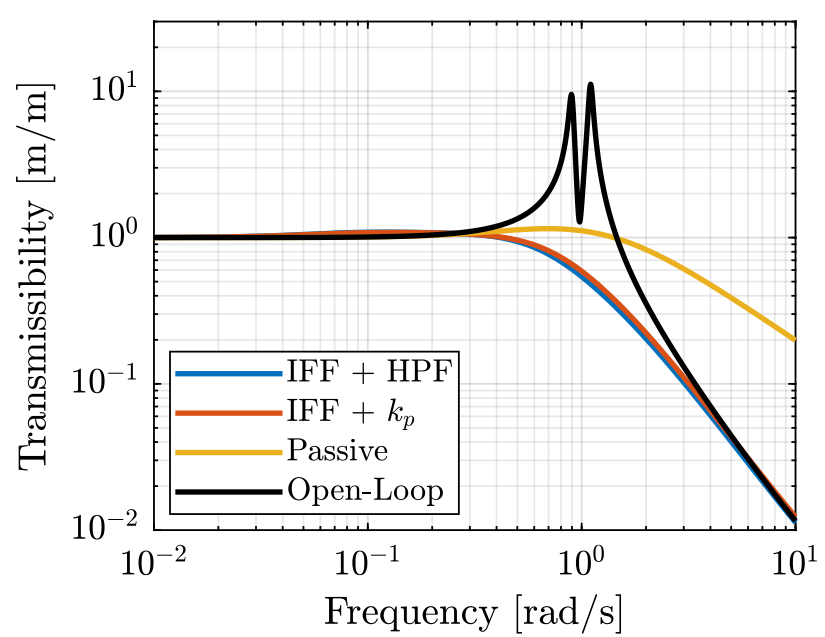

(a) Transmissibility

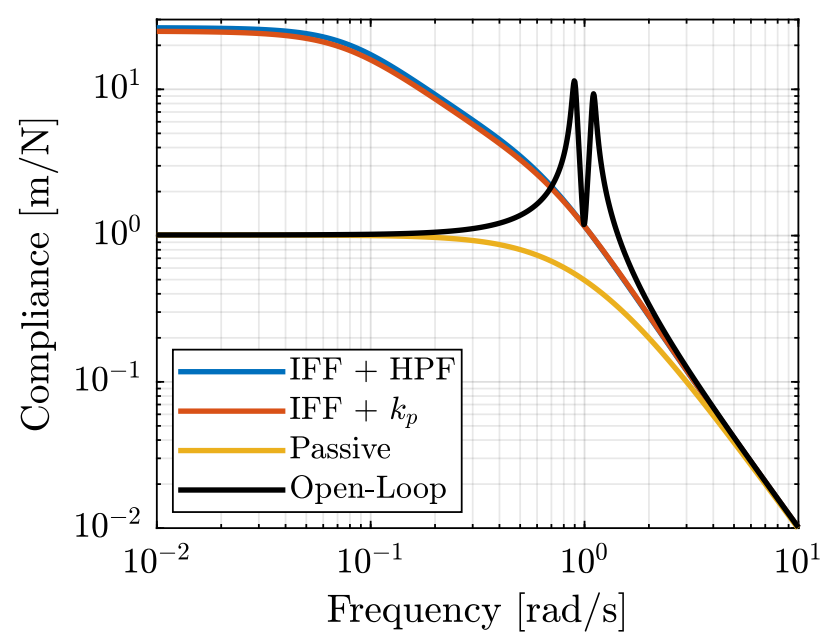

(b) Compliance

Figure 19: Comparison of the two proposed Active Damping Techniques, $\Omega=0.1 \omega_{0}$

On can see in Figure 19a that the problem of the degradation of the transmissibility at high frequency when using passive damping techniques is overcome by the use of IFF.

The addition of the HPF or the use of the parallel stiffness permit to limit the degradation of the compliance as compared with classical IFF (Figure 19b). 


\section{Conclusion}

Due to gyroscopic effects, decentralized IFF with pure integrators was shown to be unstable when applied to rotating platforms. Two modifications of the classical IFF control have been proposed to overcome this issue.

The first modification concerns the controller and consists of adding an high pass filter to the pure integrators. This is equivalent as to moving the controller pole to the left along the real axis. This renders the closed loop system stable up to some value of the controller gain $g_{\max }$.

The second proposed modification concerns the mechanical system. Additional springs are added in parallel with the actuators and force sensors. It was shown that if the stiffness $k_{p}$ of the additional springs is larger than the negative stiffness $m \Omega^{2}$ induced by centrifugal forces, the classical decentralized IFF regains its unconditional stability property.

While having very different implementations, both proposed modifications are very similar when it comes to the attainable damping and the obtained closed loop system behavior.

Future work will focus on the experimental validation of the proposed active damping techniques.

The Matlab code that was used for this study is available under a MIT License and archived in Zenodo [8].

\section{Acknowledgment}

This research benefited from a FRIA grant from the French Community of Belgium.

\section{References}

[1] A. Preumont, J.-P. Dufour, and C. Malekian, "Active damping by a local force feedback with piezoelectric actuators," Journal of guidance, control, and dynamics, vol. 15, no. 2, pp. 390-395, 1992.

[2] A. Preumont, A. François, F. Bossens, and A. Abu-Hanieh, "Force feedback versus acceleration feedback in active vibration isolation," Journal of Sound and Vibration, vol. 257, no. 4, pp. 605-613, 2002. [Online]. Available: https://doi.org/10.1006/jsvi.2002.5047

[3] A. Preumont, B. De Marneffe, and S. Krenk, "Transmission zeros in structural control with collocated multi-input/multi-output pairs," Journal of guidance, control, and dynamics, vol. 31, no. 2, pp. 428-432, 2008.

[4] S. Skogestad and I. Postlethwaite, Multivariable Feedback Control: Analysis and Design. John Wiley, 2007.

[5] A. Preumont, J.-P. Dufour, and C. Malekian, "Active damping by a local force feedback with piezoelectric actuators," in 32nd Structures, Structural Dynamics, and Materials Conference. American Institute of Aeronautics and Astronautics, apr 1991. [Online]. Available: https: //doi.org/10.2514/6.1991-989

[6] A. Souleille, T. Lampert, V. Lafarga, S. Hellegouarch, A. Rondineau, G. Rodrigues, and C. Collette, "A concept of active mount for space applications," CEAS Space Journal, vol. 10, no. 2, pp. 157-165, 2018.

[7] A. Preumont, Vibration Control of Active Structures - Fourth Edition, ser. Solid Mechanics and Its Applications. Springer International Publishing, 2018. [Online]. Available: https: //doi.org/10.1007/978-3-319-72296-2

[8] T. Dehaeze, "Active damping of rotating positioning platforms," Source Code on Zonodo, 072020. [Online]. Available: https://doi.org/10.5281/zenodo.3894342 
